\title{
Exploring the contributions of bed nets, cattle, insecticides and excitorepellency to malaria control: a deterministic model of mosquito host-seeking behaviour and mortality
}

\author{
Gerry F. Killeen ${ }^{\mathrm{a}, \mathrm{b}, \mathrm{c}, *}$, Thomas A. Smith ${ }^{\mathrm{b}}$
}

a Ifakara Health Research and Development Centre, Box 53, Ifakara, Kilombero, Morogoro, United Republic of Tanzania

b Department of Public Health and Epidemiology, Swiss Tropical Institute, Socinstrasse 57, Basel, CH 4002, Switzerland

c School of Biological and Biomedical Sciences, Durham University, South Road, Durham DH1 3LE, UK

Received 30 November 2006; received in revised form 19 April 2007; accepted 19 April 2007

Available online 13 July 2007

\section{KEYWORDS}

Malaria;

Mosquitoes;

Anopheles;

Transmission;

Ecology;

Prevention;

Host-parasite

relationship

\begin{abstract}
Summary Domestic and personal protection measures against malaria exposure either divert host-seeking vectors to other hosts or kill those attempting to feed. Here, we explicitly model mosquito host-seeking processes in the context of local host availability and elucidate the impacts and mechanisms of pyrethroid-treated bed nets in Africa. It has been suggested that excitorepellent insecticides could increase exposure of unprotected humans by concentrating mosquito biting activity on this vulnerable group. This worst-case scenario is confirmed as a possibility where vector populations lack alternative hosts, but an approximate 'break-even' scenario, with users experiencing little overall change in exposure, is more likely because of increased mosquito mortality while foraging for resources. Insecticidal nets are predicted to have epidemiologically significant impacts on transmission experienced by users and non-users at levels of coverage that can be achieved by sustainable net distribution systems, regardless of excitorepellency or the ecological setting. The results are consistent with the outcome of several randomised controlled trials, predicting enormous reductions in transmission at individual and community levels. As financial support, technology and distribution systems for insecticide-treated nets improve, massive reductions in malaria transmission could be realised. (c) 2007 Royal Society of Tropical Medicine and Hygiene. Published by Elsevier Ltd. All rights reserved.
\end{abstract}

\section{Introduction}

* Corresponding author. Tel.: +255 784477 118;

fax: +25522 2771714 .

E-mail addresses: gkilleen@ihrdc.or.tz, g.f.killeen@durham.ac.uk (G.F. Killeen).
Insecticidal measures for protection against adult mosquitoes, including treated nets and indoor residual spraying, are amongst the best established and most effec-

0035-9203/\$ - see front matter @ 2007 Royal Society of Tropical Medicine and Hygiene. Published by Elsevier Ltd. All rights reserved. doi:10.1016/j.trstmh.2007.04.022 
tive methods for the prevention of malaria (Rozendaal, 1997). The impacts of pyrethroid-treated nets (Lengeler, 2004a, 2004b) and indoor residual spraying (Kouznetsov, 1977; Mabaso et al., 2004) are clearly proven and they remain the most commonly advocated means for individuals and communities to tackle their local malaria problems (Rozendaal, 1997). The recent successes of insecticidetreated nets (ITN) have revitalised interest in vector control as a viable means to reduce malaria burden, even in parts of sub-Saharan Africa where high transmission levels result in extremely stable prevalence, incidence and clinical burden (Smith et al., 2001; Snow et al., 2005).

ITNs protect individuals either by diverting host-seeking vectors to search for a blood meal elsewhere or by killing those that attempt to feed on that person (Fanello et al., 2003; Lindsay et al., 1989, 1991, 1992; Miller et al., 1991; Pleass et al., 1993). This means that treated nets not only prevent malaria in a protected individual but can also reduce malaria risk in unprotected individuals by suppressing the density (Carnevale et al., 1988; Magesa et al., 1991; Robert and Carnevale, 1991), survival (Carnevale et al., 1988; Magesa et al., 1991; Robert and Carnevale, 1991), human blood indices (Bøgh et al., 1998; Charlwood et al., 2001) and feeding frequency (Charlwood et al., 2001) of vector populations. Conversely, it has been suggested that the excitorepellency of nets could increase the exposure of unprotected humans by concentrating the attentions of host-seeking mosquitoes upon this vulnerable portion of the population (Genton et al., 1994; Lindsay et al., 1992; Lines et al., 1987). Field studies suggest that any such inequitable effects are outweighed by beneficial impacts in whole communities (Binka et al., 1998; Gimnig et al., 2003a, 2003b; Hawley et al., 2003; Hewitt et al., 1997; Hii et al., 2001; Howard et al., 2000; Maxwell et al., 2002). Nevertheless, it is theoretically possible that interventions that divert rather than kill mosquitoes could even increase the stability of malaria transmission by increasing vectorial capacity in the most intense foci of transmission (Dye and Hasibeder, 1986; Hasibeder and Dye, 1988; Woolhouse et al., 1997).

Here we extend previously reported kinetic models of mosquito foraging for resources (Killeen et al., 2001, 2004) by explicitly modelling the processes of host seeking, encounter and attack so that the effects of bed nets and other forms of domestic protection can be explored in detail. Specifically, we investigate the likely impacts of pyrethroid-treated bed nets under conditions where holoendemic malaria is maintained by either Anopheles gambiae Giles or A. arabiensis Patton in the presence and absence of cattle as alternative hosts.

\section{Methods}

\subsection{Model framework and design strategy}

Although these principles can be extended to allow modelling of transmission by vectors with broader host ranges, here we model the feeding behaviour of the two most common malaria vectors from sub-Saharan Africa. This allows convenient simplification because these species generally feed upon only two host species: $A$. gambiae Giles and $A$. arabiensis Patton feed overwhelmingly upon either humans or cattle (Gillies and Coetzee, 1987; Gillies and DeMeillon, 1968; White, 1974). The latter feed readily upon both humans and cattle, whereas the former greatly prefer humans, particularly in East Africa (Killeen et al., 2001). Here we extend and apply recently developed deterministic models of mosquito host-choice and malaria transmission processes (Killeen et al., 2000a, 2000b, 2001, 2004) to examine the influence of vector behavioural traits and the availability of hosts upon malaria transmission intensity and the success of control measures that target adult mosquitoes. In common with almost all previous models, we assume single populations of humans, cattle and each mosquito species that interact randomly and homogeneously with no gonotrophic discordance (more than one blood meal per gonotrophic cycle (Beier, 1996)). We also assume, as demonstrated for these anthropophagic nocturnal African vector species, that host feeding success is density independent (Charlwood et al., 1995a). The conceptual basis of the model for mosquito behaviour and the effects of bed nets are outlined in Figure 1, and all symbols are defined in Table 1 for clarity and ease of reference. This model adopts a similar conditional probability approach to that previously applied to peridomestic impacts of residual insecticides (Roberts et al., 2000) and is intended to allow easier conceptualisation and parameterisation. The integration of simplified conditional probability models with kinetic (Killeen et al., 2001, 2004; Saul, 2003) and biodemographic (Carey, 2001; Killeen et al., 2000a, 2000b; Smith and McKenzie, 2004) components allows further generalisation to consider interactions with the availability of non-human hosts, diversion to unprotected humans, impacts on foraging-associated mortality and estimation of individual- and community-level impacts on human malaria exposure. New formulations, published for the first time in the paper, are outlined in the text of the Methods section, whereas reformulations of published model components are presented for reference in Appendix A.

\subsection{Model description}

As defined previously (Killeen et al., 2001), the availability (a) of any host $(j)$ of any species $(s)$ is the product of the rate at which individual vectors encounter it $\left(\varepsilon_{s, j}\right)$ and the probability that, once encountered, they will feed upon it $\left(\phi_{s, j}\right)$ :

$a_{s, j}=\varepsilon_{s, j} \phi_{s, j}$

We now consider successful feeding as just one of three possible outcomes of a host encounter by a female vector, the other two being death while attempting to feed and diversion to seek another host (Figure 1). We consider this as a two-stage process in which the vector first either attacks the encountered host or is diverted away and searches for another, the probabilities of which we denote as $\gamma_{s}$ and $\Delta_{s}$, respectively. This definition of diversion includes the combined effects of non-contact repellency and contactmediated irritancy, often referred to as excitorepellency (Muirhead-Thomson, 1960; Roberts et al., 2000). Considering mean values for hosts of any given species, the sum of 

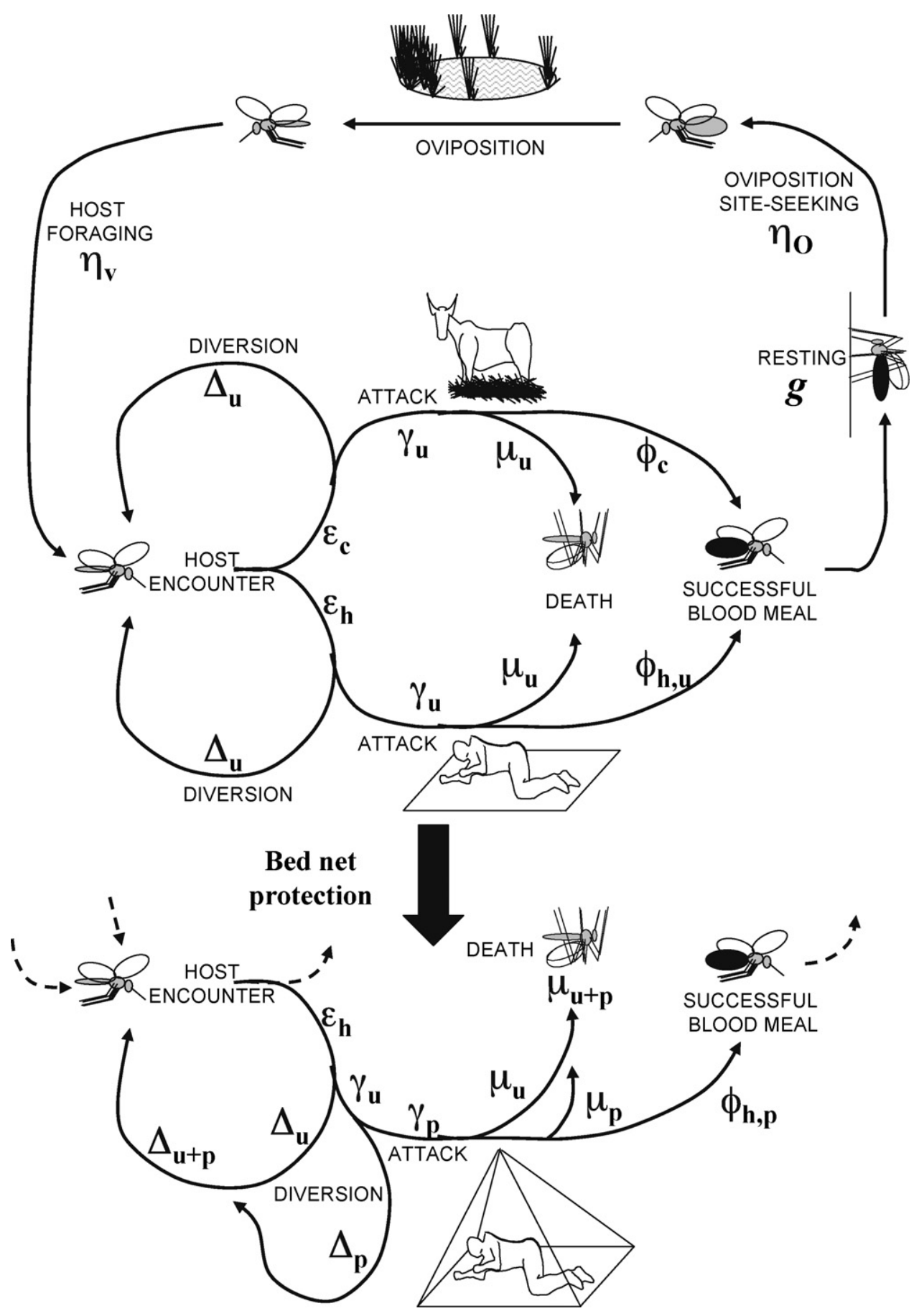

Figure 1 Diagrammatic representation of the model for the mosquito feeding cycle, outlining the rate at which mosquitoes encounter hosts $(\varepsilon)$ and the probabilities that they will attack $(\gamma)$, be diverted from $(\Delta)$, successfully feed upon $(\phi)$ or die $(\mu)$ while attempting to feed upon cattle $(c)$ or unprotected humans $(h, u)$. Also depicted are the changes to these probabilities for humans brought about by personal protection with bed nets $(p)$, resulting in overall probabilities of these events for vectors encountering protected humans $(h, p)$.

these two probabilities is:

$\gamma_{s}+\Delta_{s}=1$

We then consider that in the second stage of the blood acquisition process, namely feeding, the vector will either feed successfully or die in the attempt, the probabilities of which are denoted by $\phi_{s}$ and $\mu_{s}$, respectively. Note that $\mu_{s}$ therefore denotes the probability of death per attack rather than per encounter and this should be carefully considered when parameterising this model with field data from experimental hut trials (see Section 2.4). Thus, the probability of a successful feed per encounter is the product of two probabilities defined by losses to diversion and death: the successful feeding probability for a vector on any encounter 
Table 1 Behavioural and host availability input parameters for both vector species

\begin{tabular}{|c|c|c|c|c|c|}
\hline \multirow[t]{2}{*}{ Parameter } & \multirow[t]{2}{*}{ Unit } & \multicolumn{2}{|c|}{ Anopheles arabiensis } & \multicolumn{2}{|c|}{ Anopheles gambiae } \\
\hline & & Value & Source & Value & Source \\
\hline$\lambda_{c}$ & None & 1.6 & $\begin{array}{l}\text { Killeen et al., 2001; } \\
\text { White et al., } 1972\end{array}$ & 0.021 & $\begin{array}{l}\text { Killeen et al., 2001; } \\
\text { White et al., } 1972\end{array}$ \\
\hline$\gamma_{u}$ & $\begin{array}{l}\text { Attacks per encounter with } \\
\text { unprotected human or } \\
\text { cow }^{a, b}\end{array}$ & 0.90 & Lines et al., 1987 & 0.90 & $\begin{array}{l}\text { Assumed from Lines et } \\
\text { al. (1987) }\end{array}$ \\
\hline$\Delta_{\mathrm{u}}$ & $\begin{array}{l}\text { Attacks diverted per encounter } \\
\text { with unprotected human or } \\
\text { cow }^{\mathrm{a}, \mathrm{b}}\end{array}$ & 0.10 & $\begin{array}{l}\text { Eq. (2) and Lines et } \\
\quad \text { al., } 1987\end{array}$ & 0.10 & $\begin{array}{l}\text { Eq. (2) and assumed } \\
\text { from Lines et al. } \\
(1987)\end{array}$ \\
\hline$\Delta_{\mathrm{p}}$ & $\begin{array}{l}\text { Additional diversions per } \\
\text { protected human attacked }^{\mathrm{b}}\end{array}$ & 0.35 & Lines et al., 1987 & 0.35 & Lines et al., 1987 \\
\hline$\mu_{\mathrm{u}}$ & $\begin{array}{l}\text { Mosquito deaths per attack on } \\
\text { unprotected humans or } \\
\text { cows }^{a, b}\end{array}$ & 0.10 & Lines et al., 1987 & 0.10 & Lines et al., 1987 \\
\hline$\mu_{\mathrm{p}}$ & $\begin{array}{l}\text { Additional mosquito deaths per } \\
\text { protected human attacked }^{\mathrm{b}}\end{array}$ & 0.50 & Lines et al., 1987 & 0.50 & Lines et al., 1987 \\
\hline$a_{\mathrm{h}, \mathrm{u}}$ & $\begin{array}{l}\text { Successful feeds per day per } \\
\text { host-seeking vector per } \\
\text { unprotected human }\end{array}$ & $1.2 \times 10^{-3}$ & Killeen et al., 2004 & $1.2 \times 10^{-3}$ & Killeen et al., 2004 \\
\hline$a_{\mathrm{c}}$ & $\begin{array}{l}\text { Successful feeds per day per } \\
\text { host-seeking vector per cow }\end{array}$ & $1.5 \times 10^{-3}$ & Killeen et al., 2004 & $2.5 \times 10^{-5}$ & $=\lambda_{\mathrm{c}} a_{\mathrm{h}, \mathrm{u}}$ \\
\hline$\varepsilon_{\mathrm{h}}$ & $\begin{array}{l}\text { Encounters with humans per } \\
\text { host-seeking vector per night }\end{array}$ & $1.48 \times 10^{-3}$ & Eqs (1) and (3) & $1.48 \times 10^{-3}$ & Eqs (1) and (3) \\
\hline$\varepsilon_{\mathrm{c}}$ & $\begin{array}{l}\text { Encounters with cows per } \\
\text { host-seeking vector per night }\end{array}$ & $2.35 \times 10^{-3}$ & Eqs (1) and (3) & $3.09 \times 10^{-5}$ & Eqs (1) and (3) \\
\hline$E$ & Mosquitoes emerging per year & $9 \times 10^{6}$ & Killeen et al., 2004 & $9 \times 10^{6}$ & Killeen et al., 2004 \\
\hline$\kappa$ & $\begin{array}{l}\text { Infected mosquitoes per human } \\
\text { bite }\end{array}$ & 0.030 & Killeen et al., 2006a & 0.030 & Killeen et al., 2006a \\
\hline$N_{c}$ & Number of cattle & 0 or 1000 & Killeen et al., 2001 & 0 or 1000 & Killeen et al., 2001 \\
\hline$N_{\mathrm{h}}$ & Number of humans & 1000 & $\begin{array}{l}\text { Charlwood et al., } \\
\text { 1995a }\end{array}$ & 1000 & Charlwood et al., 1995a \\
\hline$P$ & $\begin{array}{l}\text { Proportion surviving per day } \\
\text { while resting }\end{array}$ & 0.9 & $\begin{array}{l}\text { Killeen et al., 2000b; } \\
\quad \text { Gillies, } 1954\end{array}$ & 0.9 & $\begin{array}{l}\text { Killeen et al., 2000b; } \\
\text { Gillies, } 1954\end{array}$ \\
\hline$P_{\mathrm{ov}}$ & $\begin{array}{l}\text { Proportion surviving per day } \\
\text { while foraging }\end{array}$ & $0.7-0.9$ & Saul, 2003 & $0.7-0.9$ & Saul, 2003 \\
\hline$\pi_{i}$ & $\begin{array}{l}\text { Proportion of normal human } \\
\text { exposure during which a net } \\
\text { is in use }{ }^{b}\end{array}$ & 0.90 & Killeen et al., 2006b & 0.90 & Killeen et al., 2006b \\
\hline
\end{tabular}

Detailed explanation and justification for all values and definitions are presented in the Methods section.

a Assumed to be identical for both mammalian blood sources.

b Assumed identical for both vector species.

with a host of species $s$ is the product of the probability that it will attack that host and the probability that it will survive that attack:

$\phi_{s}=\gamma_{s}\left(1-\mu_{s}\right)=\left(1-\Delta_{s}\right)\left(1-\mu_{s}\right)$

Personal protection measures such as bed nets, repellents or domestic insecticide use will modify these probabilities. We model these effects as a function of the probability that the vector would otherwise successfully feed upon a host of species $s$ (Figure 1). The effect of such a protective intervention can also be envisaged as three possible outcomes, the probabilities of which sum to 1: for a vector that would normally choose to feed upon an encountered unprotected human with a probability of $\phi_{\mathrm{h}, \mathrm{u}}$, the presence of a net or other intervention is expected to change this prob- ability to $\phi_{\mathrm{h}, \mathrm{p}}$ as a function of the additional probability of diverting $\left(\Delta_{p}\right)$ and killing $\left(\mu_{p}\right)$ that vector (Figure 1). The overall diversion $\left(\Delta_{\mathrm{u}+\mathrm{p}}\right)$ and mortality $\left(\mu_{\mathrm{u}+\mathrm{p}}\right)$ probabilities of protected hosts also depend on whether the host is actually using the net at the time, so the effect of excitorepellency and mortality is adjusted to reflect the proportion of normal exposure during which the host is actually covered $\left(\pi_{i}\right)$ :

$\Delta_{\mathrm{u}+\mathrm{p}}=\Delta_{\mathrm{u}}+\Delta_{\mathrm{p}}\left(1-\Delta_{\mathrm{u}}\right) \pi_{\mathrm{i}}$
$\mu_{\mathrm{u}+\mathrm{p}}=\mu_{\mathrm{u}}+\mu_{\mathrm{p}}\left(1-\mu_{\mathrm{u}}\right) \pi_{\mathrm{i}}$

Note that the terms $\Delta_{\mathrm{u}}$ and $\mu_{\mathrm{u}}$ are included because detailed examination of experimental hut trial results (Lines et al., 1987) indicates that a certain low but clear level of diversion does occur even in the absence of nets. This may 
be particularly important for future applications focusing on environmental management interventions that limit the availability of resources and are enhanced by increasingly lengthy foraging intervals of mosquitoes (Gu et al., 2006; Killeen et al., 2004).

For any given number of cattle $\left(N_{c}\right)$, unprotected humans $\left(N_{\mathrm{h}, \mathrm{u}}\right)$ and protected humans $\left(N_{\mathrm{h}, \mathrm{p}}\right)$, the mean seeking interval for vertebrate hosts $\left(\eta_{\mathrm{V}}\right)$ can be calculated as the reciprocal of total host availability (A) (Killeen et al., 2001), using estimates of these feeding probabilities, their corresponding encounter rates and the corresponding number of hosts of that species (s) or category $\left(A_{s}=N_{s} a_{s}\right)$ by adapting Eq. (4) of our original formulation (Killeen et al., 2001, 2004):

$\eta_{\mathrm{v}}=\frac{1}{A}=\frac{1}{A_{\mathrm{h}, \mathrm{u}}+A_{\mathrm{h}, \mathrm{p}}+A_{\mathrm{c}}}=\frac{1}{N_{\mathrm{h}, \mathrm{u}} a_{\mathrm{h}, \mathrm{u}}+N_{\mathrm{h}, \mathrm{p}} a_{\mathrm{h}, \mathrm{p}}+N_{\mathrm{c}} a_{\mathrm{c}}}$

where $A_{s}$ refers to the total availability of all hosts of species $s$. In this case, the species or species categories considered are unprotected humans $(h, u)$, protected humans $(h, p)$ and cattle (c). Values for $a_{\mathrm{c}}$ and $a_{\mathrm{h}, \mathrm{u}}$ (previously $a_{\mathrm{h}}$ ) are estimated exactly as described previously (Killeen et al., 2004; see Table 1 and Section 2.3) and $a_{\mathrm{h}, \mathrm{p}}$ is calculated as follows:

$a_{\mathrm{h}, \mathrm{p}}=\lambda_{\mathrm{p}} a_{\mathrm{h}, \mathrm{u}}$

where $\lambda_{p}$ is the relative availability of protected versus unprotected hosts, estimated in terms of the ratio of their feeding probabilities:

$\lambda_{\mathrm{p}}=\frac{\phi_{\mathrm{h}, \mathrm{p}}}{\phi_{\mathrm{h}, \mathrm{u}}}=\frac{\left(1-\Delta_{\mathrm{u}+\mathrm{p}}\right)\left(1-\mu_{\mathrm{u}+\mathrm{p}}\right)}{\left(1-\Delta_{\mathrm{u}}\right)\left(1-\mu_{\mathrm{u}}\right)}$

We adapt Eq. (3) from our previous formulation (Killeen et al., 2004) to estimate the survival rate per feeding cycle $\left(P_{f}\right)$ as the product of the probabilities of surviving the gestation $(g)$, oviposition site-seeking $\left(\eta_{0}\right)$ and vertebrate host-seeking $\left(\eta_{\mathrm{v}}\right)$ intervals, assuming a constant survival rate of $P$ for these intervals, as well as the probability of surviving the eventual attack on a host that may be protected $\left(P_{\gamma}\right)$ :

$P_{f}=P^{g+\eta 0+\eta v} P_{\gamma}$

where the mean probability of mosquitoes surviving their chosen host attack $\left(P_{\gamma}\right)$ is calculated assuming the proportion of all attacks that end in death is the sum of the mortality probabilities for attacking protected and unprotected hosts weighted according to the proportion of all encounters that will occur on such hosts. Assuming that protection does not affect encounter rates, and that these are proportional to availability when unprotected, we apply this weighting approach to estimate the total attack-related mortality rate and consequent survival as follows:

$P_{\gamma}=1-\frac{\mu_{\mathrm{u}+\mathrm{p}} a_{\mathrm{h}, \mathrm{u}} N_{\mathrm{h}, \mathrm{p}}+\mu_{\mathrm{u}}\left(a_{\mathrm{c}} N_{\mathrm{c}}+a_{\mathrm{h}, \mathrm{u}} N_{\mathrm{h}, \mathrm{u}}\right)}{a_{\mathrm{h}, \mathrm{u}}\left(N_{\mathrm{h}, \mathrm{u}}+N_{\mathrm{h}, \mathrm{p}}\right)+a_{\mathrm{c}} N_{\mathrm{c}}}$

Similarly, the human blood index is calculated as the proportion of total host availability accounted for by humans (Killeen et al., 2001), similarly to Eq. (6):

$Q_{\mathrm{h}}=\frac{A_{\mathrm{h}, \mathrm{u}}+A_{\mathrm{h}, \mathrm{p}}}{A_{\mathrm{h}, \mathrm{u}}+A_{\mathrm{h}, \mathrm{p}}+A_{\mathrm{c}}}$

The entomological inoculation rate (EIR) for protected and unprotected individuals can then be calculated from the total number of infectious bites upon humans that occur in the population as a whole ( $\beta E$; Killeen et al., 2000a, 2000b), the share of the total human availability represented by that group and the population size of that group:

$\mathrm{EIR}, \mathrm{h}=\frac{\beta E A_{\mathrm{h}, \mathrm{u}}}{A_{\mathrm{h}} N_{\mathrm{h}, \mathrm{u}}}$

$\mathrm{EIR}_{\mathrm{h}, \mathrm{p}}=\frac{\beta E A_{\mathrm{h}, \mathrm{p}}}{A_{\mathrm{h}} N_{\mathrm{h}, \mathrm{p}}}$

where $\beta$ is the mean number of infectious human bites each emerging mosquito takes in its lifetime and $E$ is the emergence rate of mosquitoes (Killeen et al., 2000b, 2004). Dividing Eq. (13) by Eq. (12), substituting with Eq. (7) and rearranging also leads to an intuitively satisfactory solution:

$E I R_{h, p}=\lambda_{p} E I R_{h, u}$

Otherwise, malaria transmission is modelled exactly as described previously (Killeen et al., 2004). This model was adapted from its original formulation (Killeen et al., 2000b) to account for superinfection of mosquitoes (Smith and McKenzie, 2004) and to smooth the effects of changing host availability patterns on feeding cycle length (Killeen et al., 2004). Specifically, the model is adapted to a daily cycle and cumulative survival up to each age $(x)$ is estimated as follows and used to calculate the EIR and associated parameters as previously described (Killeen et al., 2000b, 2004):

$P_{x}=P_{f}^{x / f}$

where $f$ is the mean feeding cycle length of the vector population. Similarly, the sporozoite infection prevalence of mosquitoes at each age is considered in days, accounting for superinfection:

$S_{x}=S_{x-1}+\frac{\kappa Q\left(1-S_{x-1}\right)}{f}$, where $x>n$, otherwise $S_{i}=0$

where $\kappa$ denotes the mean infectiousness of the human population to vector mosquitoes (Killeen et al., 2006a) and $n$ is the duration of the sporogonic development period of the parasite from ingestion to infective sporozoite stages (Killeen et al., 2000a). Survival and infectiveness probabilities are calculated up to 40 days, after which the contributions of mosquitoes in these age classes to transmission become negligible. Note that $P_{x}$ is multiplied by $S_{x}$ to obtain the corresponding probability of being both alive and infective $\left(I_{x}\right)$ on each day, and the relevant mosquito lifetime biodemographic parameters required to predict the EIR are calculated by summing these three age-specific outcomes as previously described (Killeen et al., 2000b, 2004).

\subsection{Baseline mosquito behaviour, host availability and survival parameters}

As an example, we take Namawala in the Kilombero Valley, Tanzania, as a primary centre for parameterising our model because of the exceptionally detailed quantitative characterisation of malaria transmission and vector biodemography in this village and the surrounding area. This is a holoendemic village with intense seasonal transmission, stable high parasite prevalence in humans and a heavy burden of clinical malaria (Charlwood et al., 1995a, 1995b, 1997, 
1998; Kitua et al., 1996; Smith et al., 1993, 1995, 1998). This is a site where the bulk of transmission is mediated by A. gambiae s.l. (of which the main species involved in transmission is $A$. arabiensis) and where transmission intensity has been modelled with available field data (Killeen et al., 2000a, 2000b).

As previously (Killeen et al., 2000a, 2000b), we base our estimate of human population size (Charlwood et al., 1995a) approximately upon those reported for this particular village during the early 1990s. Nevertheless, we use a human population size of 1000 and, where relevant, a bovine population of the same size (Killeen et al., 2001) so that the EIR experienced by users and non-users can be easily calculated at net coverage levels approaching $0 \%$ and $100 \%$. By setting coverage to 0.001 or 0.999 , this simulates a single user or non-user in the population, respectively. Infectiousness of humans $(\kappa)$ is set to 0.030 , reflecting a more precise recent estimate (Killeen et al., 2006a) than was available previously (Charlwood et al., 1995b, 1997). The emergence rate remains set at $9 \times 10^{6}$ emerging vectors per year, as previously described (Killeen et al., 2004). We set mean daily survival of hazards other than feeding $(P)$ at 0.90 , reflecting a median value of daily survival at four well characterised holoendemic sites (Killeen et al., 2000b) and estimated daily indoor survival for A. gambiae s.l. in Tanzania (Gillies, 1954). The results of experimental hut studies (Lines et al., 1987) are combined with host-choice evaluations (White et al., 1972) and appropriate analytical models (Killeen et al., $2001,2004)$ to define the attack and mortality probabilities of $A$. arabiensis encountering cattle or humans: we set the probability that $A$. arabiensis will attack unprotected cattle or humans $\left(\gamma_{\mathrm{u}}\right)$, conditional upon encountering them, to be 0.90 and the chance that they will die in the attempt $\left(\mu_{\mathrm{u}}\right)$ at 0.10 . Using these parameters and Eq. (3), we calculate that for $A$. arabiensis the overall feeding probability upon either cattle $\left(\phi_{\mathrm{c}}\right)$ or unprotected humans $\left(\phi_{\mathrm{h}}\right)$ would be 0.81 , a value similar to previous estimates of approximately $0.80-0.85$ for the feeding success of $A$. gambiae s.l. upon sleeping humans in Tanzania (Charlwood et al., 1995a; Lines et al., 1987). We also apply these same probabilities of attacking $\left(\gamma_{\mathrm{u}}\right)$, feeding $\left(\phi_{\mathrm{h}, \mathrm{u}}\right)$ and dying $\left(\mu_{\mathrm{u}}\right)$ to $A$. gambiae s.s. encountering unprotected humans. The availabilities of unprotected humans and cattle are calculated for $A$. arabiensis using field measurements of the duration of the feeding cycle and extended to $A$. gambiae, accounting for the lower estimated relative availability of cattle $\left(\lambda_{c}\right)$ to this mosquito species as previously described (Killeen et al., 2001; Table 1). Note that $\lambda_{\mathrm{c}}$ is assumed to modify $a_{\mathrm{c}}$ by affecting the encounter rate only, indicating that these mosquitoes can differentiate between preferred and nonpreferred hosts at long range (Gillies and Wilkes, 1969, 1970, 1972). In the case of $A$. arabiensis, this assumption is consistent with the longer range of attraction of cows relative to humans for zoophilic members of the $A$. gambiae complex (Gillies and Wilkes, 1969, 1970, 1972).

\subsection{Parameters reflecting the effects of insecticide-treated bed nets}

The effects of ITNs upon feeding probability and mortality risk of either $A$. gambiae s.l. sibling species encountering a protected human are assumed to be identical and are derived from the results of detailed experimental hut trials from northern Tanzania (Lines et al., 1987). Trials with A. arabiensis were carried out in Magugu, west of Arusha, under experimental conditions that excluded cattle from the immediate surroundings, and with $A$. gambiae in Muheza, near Tanga. In this study, nylon nets were impregnated with $0.2 \mathrm{~g} / \mathrm{m}^{2}$ permethrin and their effects on house entry, feeding success and survival were quantified. Combining results from nets with and without holes, these authors reported that, of vectors that would otherwise feed successfully, the proportion that successfully fed and survived the hazards of the treated net $\left(\phi_{p}\right)$ was approximately $0.11-0.17$. This reduced feeding and survival success was attributed to an increased mortality $\left(\mu_{p}\right)$ of approximately $0.50-0.55$, implying that the proportion of vectors being diverted by the nets $\left(\Delta_{\mathrm{p}}\right)$ was approximately $0.27-0.38$. Based on these estimates, we set $\mu_{\mathrm{p}}$ and $\Delta_{\mathrm{p}}$ at 0.50 and 0.35 , respectively (Table 1). Although the ability of permethrin to divert or kill vectors varies considerably with formulation (Lindsay et al., 1991; Pleass et al., 1993), the values we have chosen compare well with those from other studies applying similar permethrin doses in East and West Africa (Mathenge et al., 2001; Pleass et al., 1993). The proportion of normal biting exposure that occurs while nets are actually in use $\left(\pi_{i}\right)$ has been estimated as $90 \%$ for $A$. gambiae in southern Tanzania (Killeen et al., 2006b), so we set $\pi_{i}$ to a value of 0.90 . Note that unlike previously published applications of this model (Killeen et al., 2006b), here we use $\pi_{i}$ rather than $\pi_{\mathrm{s}}$, the proportion of bites that occur during peak sleeping hours, because the former more comprehensively captures the level of protection afforded by a net.

\subsection{Testing the sensitivity of the conclusions to increased mosquito mortality while foraging for resources}

Foraging for resources is an intrinsically dangerous undertaking for mosquitoes and it is almost certain that survival during these phases is lower than while resting in houses (Kelly and Thompson, 2000; Saul, 2003). Assuming this is the case, the simulations we have outlined thus far will underestimate the impact of diversionary measures for non-users because these interventions are expected to have a larger impact on mosquito survival, sporozoite prevalence and EIR if foraging is more hazardous than resting. We therefore tested the sensitivity of our conclusions to deviations from the assumption that mortality is constant across all phases of the life cycle. This was accomplished by separately considering survival of the resting $(P)$, foraging $\left(P_{\text {ov }}\right)$ and attacking $\left(P_{\gamma}\right)$ phases and adapting Eq. (9) accordingly:

$P_{f}=P^{g} P_{\mathrm{ov}}^{\eta 0+\eta v} P_{\gamma}$

where $P_{\text {ov }}$ is an assumed common survival rate for mosquitoes foraging for either oviposition sites or vertebrate hosts. Values for $P_{\text {ov }}$ were varied from 0.9 (equivalent to the formulation described above in Eq. (9)) down to 0.7 , reflecting a potentially realistic range of foraging survival rates under field conditions (Saul, 2003). This range of $P_{\text {ov }}$ was evaluated in terms of its impact on the protection afforded to unprotected individuals in populations 
where $75 \%$ of the human population used nets that diverted, killed, or diverted and killed mosquitoes. This analysis was conducted for both of the two distinct scenarios described above: A. gambiae s.l. in the absence of alternative hosts and $A$. arabiensis in the presence of cattle.
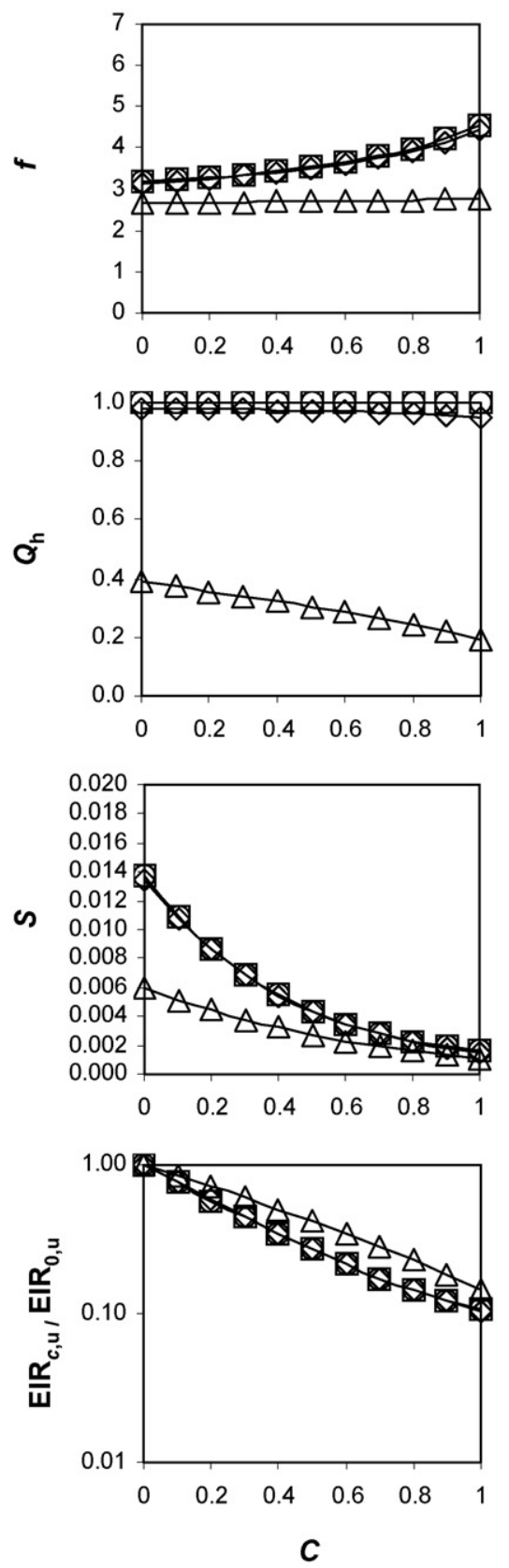

\section{Results}

First we compare the predicted effects of increasing coverage of effectively-treated nets with both diversionary and insecticidal properties in four different scenarios: pure
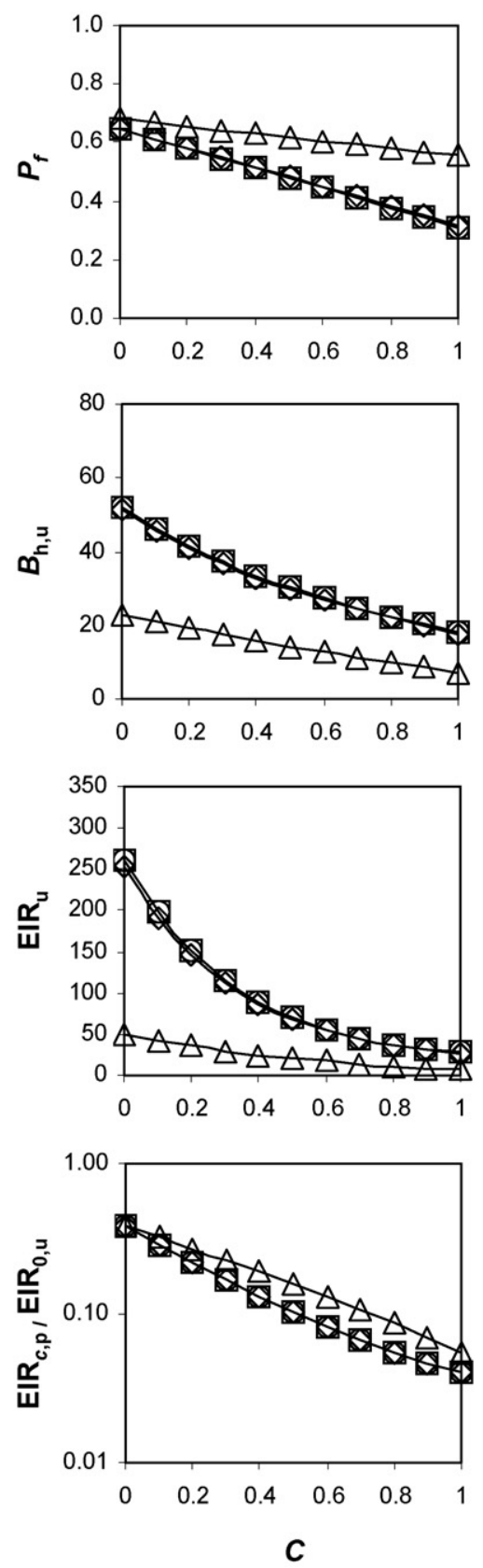

Figure 2 Predicted effects upon malaria transmission intensity of insecticide-treated nets that both divert and kill mosquitoes. The diversionary and insecticidal properties of the nets are as described in experimental hut trials (Lines et al., 1987) and are summarised in Section 2.4. The outcome variables plotted on the $y$-axes are the survival probability per feeding cycle $\left(P_{f}\right.$; Eq. (9)), the human blood index $\left(Q_{h}\right.$; Eq. (11); human bites per bite), the feeding cycle length ( $f$; Eq. (A.1); nights), the biting rate experienced by unprotected humans ( $B_{\mathrm{h}, \mathrm{u}}$; Eq. (A.5); bites per person per night), the sporozoite prevalence ( $S$; Eq. (A.4); infectious bites per bite), the entomological inoculation rate of an unprotected human (EIR ; Eq. (12); infectious bites per person per year) and the relative exposure of unprotected community members $\left(\operatorname{EIR}_{c, \mathrm{u}} / \mathrm{EIR}_{0, \mathrm{u}}\right.$; Eq. (12)) as well as protected members using nets $\left(E_{c, p} / R / R_{0, u}\right.$; Eqs (12) and (13)). These outcomes are plotted as a function of increasing levels of coverage with effectively treated nets ( $C$, expressed in terms of net use) for Anopheles gambiae and $A$. arabiensis vector populations in the presence and absence of one head of cattle per person: A. arabiensis without cattle ( $\square)$, A. arabiensis with cattle $(\triangle)$, A. gambiae s.s. without cattle $(\bigcirc)$ and $A$. gambiae s.s. with cattle $(\diamond)$. 
populations of either $A$. arabiensis or $A$. gambiae s.s. in the presence or absence of cattle (Figure 2 ).

The individual protection afforded to users is constant for all scenarios and coverage levels because this component of protection only occurs in and around the domestic environment where the parameter values, as well as the entities and processes they describe, are assumed identical. Nets are predicted to prevent $57 \%$ of exposure amongst users. This is somewhat lower than previous field estimates of $69 \%$ at a northern Tanzanian site very close to some of those used to parameterise this model (Soremekun et al., 2004). Although this difference might be partially explained by the modest excitorepellent and insecticidal properties we have assumed for treated nets, complementary behavioural studies indicate approximately $10 \%$ of normal exposure occurs outdoors (Killeen et al., 2006b). The inability of nets to prevent this directly is unlikely to be captured by the estimates of Soremekun et al. (2004) because only mosquitoes that entered houses were sampled. Thus, our simulation of protection against all exposure appears quite compatible with the estimate of Soremekun et al. (2004), which probably reflects protection against indoor exposure only.

Next, we go beyond the direct protection afforded to individual users to consider community-level protection through impacts on vector-parasite biodemography (Figure 2). The presence of cattle has a substantial influence on the effects of nets only for $A$. arabiensis, so all subsequent comparisons consider only two scenarios: (1) a population of either species ( $A$. gambiae s.l.) in the absence of cattle; and (2) an A. arabiensis population in the presence of cattle. In all scenarios described in Figure 2, ITNs reduce transmission both for users and non-users by lowering mosquito survival. Nets also act by extending the length of the mosquito feeding cycle in all scenarios, except for A. arabiensis in the presence of cattle. In this case, however, the failure to increase feeding cycle length results from diversion of mosquitoes to cattle so that the human blood index, and hence transmission intensity, is reduced. The availability of cattle as an alternative host to A. arabiensis does somewhat reduce the impact of nets on mosquito survival and EIR. In all cases, however, epidemiologically significant impacts on EIR of users and, to a lesser extent, non-users are predicted at levels of coverage that have already been achieved through established delivery mechanisms. As proposed based on large-scale field trials (Hawley et al., 2003), absolute coverage of $50 \%$ use of effectivelytreated nets is expected to achieve useful community-wide protection of non-users in all scenarios, and increasing gains are realised as coverage is increased further. Specifically, the lowest predicted level of protection against exposure was for non-users and $A$. arabiensis in the presence of cattle, but even this $58 \%$ reduction at $50 \%$ coverage closely approaches the $60-70 \%$ reductions thought to account for the personal protection of ITNs against malarial disease, regardless of the local endemicity level (Killeen et al., 2006b; Soremekun et al., 2004). We note that sustained large-scale net distribution systems in the Kilombero Valley, southern Tanzania, have exceeded this target and achieved $75 \%$ net use (Killeen et al., unpublished data) using hybrid social marketing systems that deliver targeted public sector subsidies for ITNs that are obtained through the private sector (Magesa et al., 2005; Mushi et al., 2003; Schellenberg et al., 2001).
In the second set of scenarios, we explore the impacts of nets with varying levels of diversionary and insecticidal properties upon the EIR (Figure 3) at a coverage rate of $75 \%$, consistent with recent programmatic observations in southern Tanzania (Killeen et al., unpublished data). All formulations confer protection to users and non-users alike for A. arabiensis in the presence of cattle. However, the use of excitorepellent formulations in the absence of cattle actually appears to increase the malaria transmission intensity experienced by non-users, regardless of the vector species or insecticidal properties of the net. Thus, the worst-case scenario (Genton et al., 1994; Lindsay et al., 1992; Lines et al., 1987), in which the impacts of excitorepellency on mosquito survival, feeding cycle length and human feeding frequency are outweighed by the concentration of remaining transmission upon the most vulnerable, appears to be possible under these conditions. However, this does not appear to be a realistic scenario given the proven impact of ITNs for non-users regardless of the degree of anthropophily of local vector populations (Binka et al., 1998; Gimnig et al., 2003a, 2003b; Hawley et al., 2003; Hewitt et al., 1997; Hii et al., 2001; Howard et al., 2000; Maxwell et al., 2002). Examining the simulated situation more closely, the assumption of constant mosquito mortality throughout the gonotrophic cycle is crucial and almost certainly underestimates the impact of diversion on mosquito longevity and community-level transmission for users and non-users alike (Kelly and Thompson, 2000; Saul, 2003).

We therefore tested the sensitivity of our predictions to increased mosquito mortality during the active foraging phase of the host-seeking interval. Figure 4 reveals just how insensitive the efficacies of all formulations are to more realistic levels of foraging-associated mortality $\left(P_{\mathrm{ov}}\right)$. The communal protection afforded to non-users in populations with high coverage of ITNs is completely insensitive to $P_{\text {ov }}$ for all nets that are insecticidal, regardless of the vector system. Protection of non-users by purely excitorepellent ITNs is minimal and similarly insensitive to $P_{\mathrm{ov}}$ for human populations exposed to zoophilic vectors such as $A$. arabiensis in the presence of alternative hosts. For human-dependent $A$. gambiae in the absence of alternative hosts, very low values of $P_{\mathrm{ov}}$ are required to kill diverted mosquitoes fast enough to prevent increased risk among non-users. If the mosquito survival rate while foraging approaches that measured while resting indoors, purely excitorepellent nets could increase the exposure of unprotected humans by concentrating the attentions of host-seeking mosquitoes upon this vulnerable portion of the population (Genton et al., 1994; Lindsay et al., 1992; Lines et al., 1987). Such an eventuality could even increase the stability of malaria transmission by increasing vectorial capacity in the most intense foci of transmission (Dye and Hasibeder, 1986; Hasibeder and Dye, 1988; Woolhouse et al., 1997). Whilst we suggest that such high survival rates for foraging mosquitoes are highly unlikely (Kelly and Thompson, 2000; Saul, 2003), setting lower $P_{\text {ov }}$ values suggests an approximately break-even outcome for non-users where vector populations lack alternative hosts. Below the median plausible foraging survival rate of 0.8 per day, high coverage with ITNs results in higher biting rates upon nonusers but lower sporozoite prevalence so that no substantial change of EIR is expected. 
Anopheles gambiae s.I. in the absence of cattle
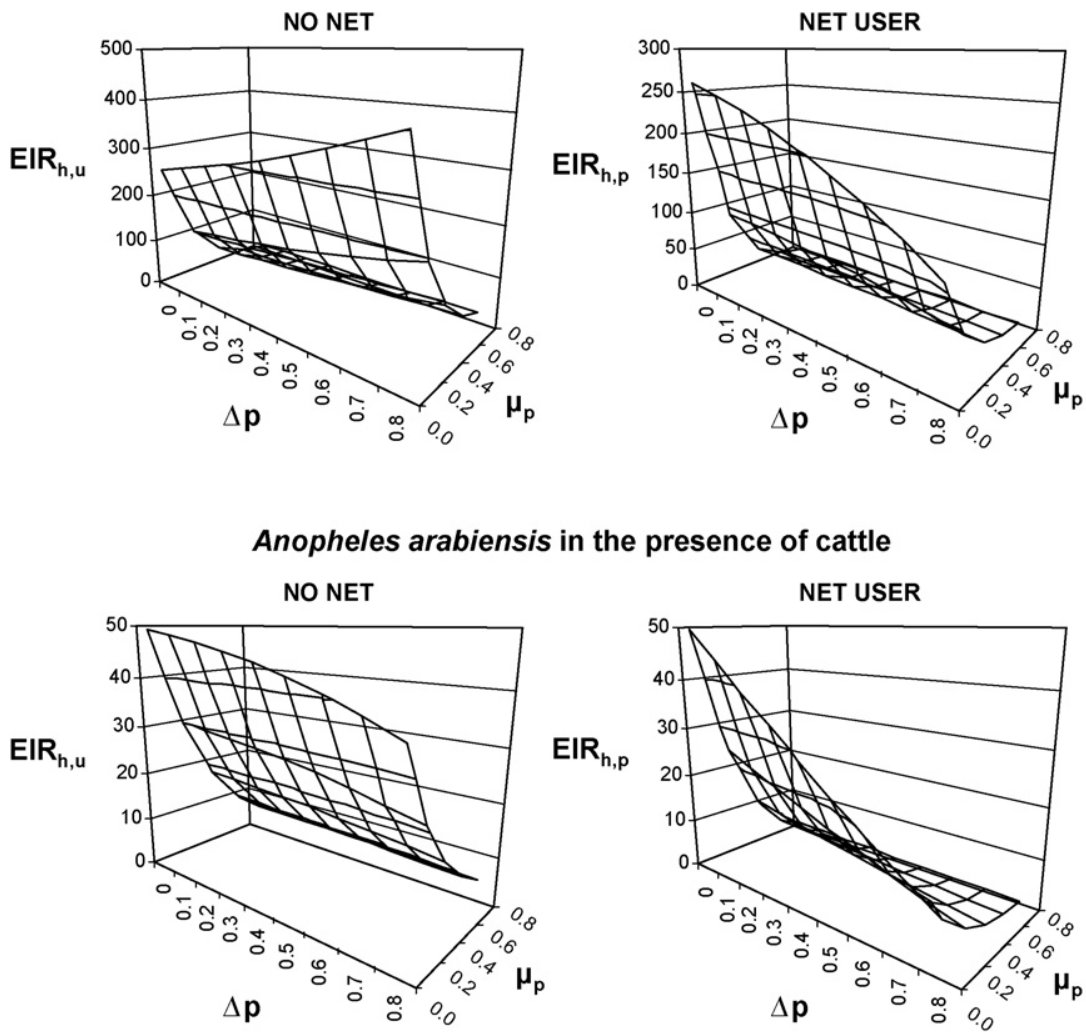

Figure 3 Impacts of insecticide-treated nets on malaria transmission as a function of their ability to divert and kill host-seeking mosquitoes. Malaria transmission intensity (entomological inoculation rate) for individuals with (EIR $\mathrm{h}_{\mathrm{p}}$; Eq. (13)) and without $\left(\mathrm{EIR}_{\mathrm{h}, \mathrm{u}}\right.$; Eq. (12)) nets is plotted as a function of their ability to divert $\left(\Delta_{p}\right)$ and kill $\left(\mu_{p}\right)$ mosquitoes attacking protected humans. The results presented represent simulations assuming $75 \%$ usage of nets in two distinctive scenarios: Anopheles gambiae s.l. in the absence of cattle (results for both sibling species are identical) and $A$. arabiensis in the presence of one head of cattle per person.

Figure 5 illustrates this point more succinctly. All combinations of excitorepellency and insecticidal properties will protect users against exposure, but those lacking insecticidal properties will provide little or no communal protection and will therefore fail to protect non-users. Excitorepellency will always enhance the protection of ITN users and is expected to improve protection of non-users where alternative hosts are available but to slightly attenuate this benefit
A. gambiae s.I. in the absence of cattle

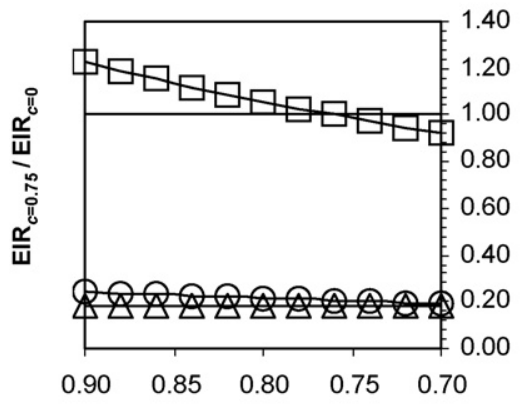

A. arabiensis in the presence of cattle

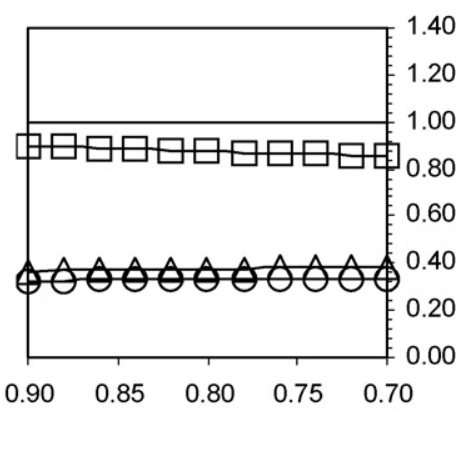

Figure 4 Influence of reduced mosquito survival during foraging ( $P_{\text {ov }}$; Eq. (17)) on the protection of non-users (Eq. (12)) by nets that divert $(\square)$, kill $(\triangle)$ or divert and kill $(\bigcirc)$ mosquitoes. We assume $75 \%$ coverage with nets $(C=0.75$; Killeen et al., unpublished data) that cause $40 \%$ diversion $\left(\Delta_{p}=0.4\right)$ and/or mortality $\left(\mu_{p}=0.4\right)$ of mosquitoes in two scenarios: Anopheles gambiae s.l. in the absence of alternative hosts and $A$. arabiensis in the presence of cattle. Protection is expressed in terms of reduction in the entomological inoculation rate (EIR) relative to conditions without any nets $(C=0)$ in the community at the same value of survival during foraging $\left(P_{\mathrm{ov}}\right)$. 


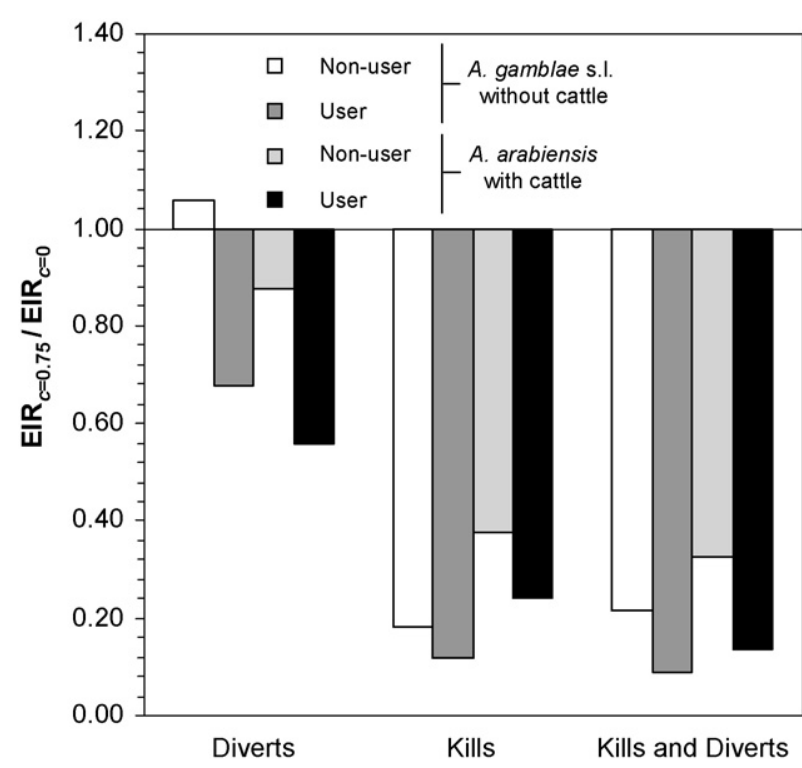

Figure 5 Impact of nets that divert, kill, or divert and kill mosquitoes on transmission intensity experienced by users and non-users (Eqs (12) and (13)). We assume 75\% coverage with nets (Killeen et al., unpublished data) that cause $40 \%$ diversion $\left(\Delta_{p}=0.4\right)$ and/or mortality $\left(\mu_{p}=0.4\right)$ in two distinct scenarios: Anopheles gambiae s.l. in the absence of alternative hosts and A. arabiensis in the presence of cattle. Impact is expressed in terms of reduction in the entomological inoculation rate (EIR) relative to conditions without any nets $(C=0)$ elsewhere in the community. Mosquito survival while foraging $\left(P_{\text {ov }}\right.$; Eq. $\left.(17)\right)$ was set to a median plausible value of 0.80 per day.

where humans are the only available host. We nevertheless suggest that excitorepellency will improve the effectiveness of insecticidal ITNs, even in the latter scenario, because this will enhance the personal protection that motivates individual uptake and subsequently increases community-level coverage.

\section{Discussion}

Here we have outlined a kinetic mosquito behaviour and mortality model, parameterised it with field measurements of its component processes, and predicted impacts on malaria transmission that are consistent with the results of large-scale randomised controlled trials. Extending these results to consider vector populations that are resistant to the insecticidal properties of net treatments but not their excitorepellent properties (Potikasikorn et al., 2005) supports the view that treated nets can provide personal protection even when they fail to kill target mosquito species (Fanello et al., 2003). Nevertheless, the model suggests that purely diversionary interventions will probably have minimal impacts on community-level transmission and the exposure of non-users. The worst-case scenario in which high coverage with purely excitorepellent interventions increases exposure of non-users is theoretically unlikely but not impossible. In practice, it has been observed that pyrethroid-treated ITNs can even benefit non-users where $k d r$ resistance alleles occur at high frequencies (Henry et al., 2005), although it should be noted that West African A. gambiae may be more zoophilic than the East African populations used to parameterise this model (Killeen et al., 2001). We therefore suggest that operational programmes delivering domestic interventions such as ITNs or indoor residual sprays should prioritise products based on their insecticidal properties but also opt for formulations that maximise excitorepellency and personal protection.

This model and argument could just as easily be applied to indoor residual spraying of dichlorodiphenyltrichloroethane (DDT), a comparably efficacious intervention (Curtis and Mnzava, 2000) with similar impacts upon the survival of $A$. gambiae (Magesa et al., 1991). Roberts et al. (2000) consider that DDT functions effectively, even against resistant zoophagic vector populations in Thailand, India and Mexico (Potikasikorn et al., 2005; Roberts and Andre, 1994), because it acts largely through excitorepellent diversion rather than direct killing. Thus, the approach described here may be particularly useful for elucidating the mechanisms and effects of such largely diversionary intervention strategies. Unlike previous models based on experimental hut studies alone (Roberts et al., 2000), the survival cost of extended dispersal periods outside of houses can be simulated and its contribution to the overall impact can be predicted in quantitative terms. This model may also be developed to assess alternative strategies such as insecticide treatment of cattle (Kawaguchi et al., 2004; Rowland et al., 2001; Saul, 2003). Although irrelevant in some situations (Charlwood et al., 1995a), density-dependent feeding success has been documented for these vectors (Lindsay et al., 1995) and this potential complication should be considered before extending to other host-vector systems (Kelly and Thompson, 2000). It should be noted, however, that the kind of excitorepellency we simulate here is a long-lasting property of the net and similar impacts are unlikely to be achieved through the application of volatile substances such as diethyltoluamide (DEET) (Pennetier et al., 2005) because these formulations were found to have only a short persistence when applied to bed nets (Curtis et al., 1987).

Unfortunately, most nets in Africa have, until recently, been non-insecticidal in practice (Roll Back Malaria Partnership, 2005a) and typically in poor condition with many holes under normal conditions of use (Erlanger et al., 2004; Maxwell et al., 2006a). Untreated nets under typical field conditions (Erlanger et al., 2004; Maxwell et al., 2006a) confer little, if any, protection (Curtis et al., 1996; Lines et al., 1987), so national programmes throughout Africa should now focus on achieving high coverage of target groups with nets that remain repellent and insecticidal throughout their lifetime (Roll Back Malaria Partnership, 2005b). This can be achieved by distributing long-lasting insecticidal nets or by applying long-lasting insecticide formulations to existing nets that would otherwise remain ineffective (Asidi et al., 2004, 2005; Curtis et al., 1996; Graham et al., 2005; Maxwell et al., 2006b; Tami et al., 2004; Yates et al., 2005). Many of the simulations described here have been based on conservative estimates of the insecticidal and diversionary properties of nets, consistent with modest levels of treatment under operational conditions and with treatment technologies as they were two decades ago. Today, long-lasting insecticide treatments with much better insecticidal properties (Asidi et al., 2004, 2005; Curtis et 
al., 1996; Graham et al., 2005; Maxwell et al., 2006b; Yates et al., 2005) that are retained for up to 7 years of normal use (Tami et al., 2004) as well as durable net materials that that can last up to 15 years (Killeen, unpublished data) are becoming a proven reality.

Whilst the choice of delivery strategy for ITNs has been contentious in recent years (Curtis et al., 2003; Lines et al., 2003), consensus is emerging that public sector (Anonymous, 2006; Grabowsky et al., 2005) and marketbased approaches (Lines et al., 2003; Webster et al., 2005) as well as hybrid systems (Magesa et al., 2005; Mushi et al., 2003) merit investigation, development and long-term evaluation on scales for which no precedent yet exists (Roll Back Malaria Partnership, 2005b). Programmatic-scale evaluations in southern Tanzania suggest hybrid social marketing systems, which combine the strengths of both public sector and market-based systems (Magesa et al., 2005; Mushi et al., 2003; Schellenberg et al., 2001) to achieve high population-level coverage with nets (Killeen et al., unpublished data), could be greatly enhanced by these exciting new ITN technologies. If these same levels of coverage can be achieved with long-lasting insecticidal nets rather than ordinary, poorly maintained and largely untreated nets (Erlanger et al., 2004; Killeen et al., unpublished data; Maxwell et al., 2006a), it may soon be possible to realise the massive malaria transmission reductions predicted under the ideal conditions presented in the right-hand corner of each panel in Figure 3. As global support for malaria control in Africa continues to grow (Haines and Cassels, 2004; Millennium Development Project, 2005) and scale-up of ITN use proceeds across Africa (Roll Back Malaria Partnership, 2005a, 2005b), it should be possible to translate theory into practice.

Authors' contributions: GFK conceived the model and then developed and applied it with assistance from TAS. GFK drafted the manuscript in consultation with TAS and both authors read and approved the final version. GFK and TAS are guarantors of the paper.

Acknowledgement: We thank two anonymous referees for their meticulous and constructive comments on this manuscript.

Funding: Swiss National Science Foundation (award number 2200C0-105994) and the Bill and Melinda Gates Foundation (award number 39777) are thanked for financial support. GFK is supported by the Wellcome Trust through a Research Career Development Fellowship (award number 076806).

Conflicts of interest: None declared.

Ethical approval: Not required.

\section{Appendix A}

Here, we briefly outline previously described equations using symbols and terminology revised in accordance with those presented in the Methods section of this paper. The feeding cycle length $(f)$ is calculated as the sum of the durations of the gestation period $(g)$, the oviposition site-seeking interval $\left(\eta_{\mathrm{o}}\right)$ and the vertebrate host-seeking interval $\left(\eta_{\mathrm{v}}\right)$ :

$f=g+\eta_{\mathrm{o}}+\eta_{\mathrm{v}}$

Adapting Eq. (2) from Killeen et al. (2000b) to the nightly time increment described in Eq. (15), the number of human bites the average mosquito takes in a lifetime $\left(b_{h}\right)$ is defined as the sum of the probabilities of surviving and feeding on a human at each age $(x)$ :

$b_{\mathrm{h}}=Q_{\mathrm{h}} \sum_{x}^{\infty} \frac{P_{\mathrm{f}}^{\mathrm{x} / \mathrm{f}}}{f}$

Similarly, accounting for superinfection as described in Eq. (16), the number of infectious bites on humans per mosquito lifetime $(\beta)$ is calculated as the product of the human blood index and sum of the products of the probabilities of biting and being infectious at each age (Killeen et al., 2000b):

$\beta=Q_{\mathrm{h}} \sum_{\mathrm{x}}^{\infty} \mathrm{S}_{\mathrm{x}} \frac{P_{\mathrm{f}}^{\mathrm{x} / \mathrm{f}}}{f}$

The overall sporozoite prevalence can then be calculated as the quotient of $b_{\mathrm{h}}$ and $\beta$ (Killeen et al., 2000b; Eq. (14)):

$S=\frac{b_{\mathrm{h}}}{\beta}$

Replacing $\beta$ with $b_{\mathrm{h}}$ in Eq. (12), the biting rate experienced by unprotected $\left(B_{\mathrm{h}, \mathrm{u}}\right)$ and protected humans $\left(B_{\mathrm{h}, \mathrm{p}}\right)$ is calculated as previously described (Killeen et al., 2000b):

$$
\begin{aligned}
B_{\mathrm{h}, \mathrm{u}} & =\frac{b_{\mathrm{h}} E A_{\mathrm{h}, \mathrm{u}}}{A_{\mathrm{h}} N_{\mathrm{h}, \mathrm{u}}} \\
B_{\mathrm{h}, \mathrm{p}} & =\frac{b_{\mathrm{h}} E A_{\mathrm{h}, \mathrm{p}}}{A_{\mathrm{h}} N_{\mathrm{h}, \mathrm{p}}}
\end{aligned}
$$

\section{References}

Anonymous, 2006. Distribution of insecticide-treated nets during a polio immunization campaign in Niger. MMWR Morb. Mortal. Wkly Rep. 55, 913-916.

Asidi, A.N., N'Guessan, R., Hutchinson, R.A., Traore-Lamizana, M., Carnevale, P., Curtis, C.F., 2004. Experimental hut comparisons of nets treated with carbamate or pyrethroid insecticides, washed or unwashed, against pyrethroid-resistant mosquitoes. Med. Vet. Entomol. 18, 134-140.

Asidi, A.N., N'Guessan, R., Koffi, A.A., Curtis, C.F., Hougard, J.M., Chandre, F., Corbel, V., Darriet, F., Zaim, M., Rowland, M.W., 2005. Experimental hut evaluation of bednets treated with an organophosphate (chlorpyrifos-methyl) or a pyrethroid (lambdacyhalothrin) alone and in combination against insecticide-resistant Anopheles gambiae and Culex quinquefasciatus mosquitoes. Malar. J. 4, 25.

Beier, J.C., 1996. Frequent blood-feeding and restrictive sugarfeeding behavior enhance the malaria vector potential of Anopheles gambiae s.l. and An. funestus (Diptera: Culicidae) in western Kenya. J. Med. Entomol. 33, 613-618.

Binka, F.N., Indome, F., Smith, T., 1998. Impact of spatial distribution of permethrin-impregnated bed nets on child mortality in rural northern Ghana. Am. J. Trop. Med. Hyg. 59, 80-85.

Bøgh, C., Pedersen, E.M., Mukoko, D.A., Ouma, J.H., 1998. Permethrin-impregnated bed net effects on resting and feed- 
ing behaviour of lymphatic filariasis vector mosquitoes in Kenya. Med. Vet. Entomol. 12, 52-59.

Carey, J.R., 2001. Insect biodemography. Ann. Rev. Entomol. 46, 79-110.

Carnevale, P., Robert, V., Boudin, C., Halna, J.M., Pazart, L., Gazin, P., Richard, A., Mouchet, J., 1988. Control of malaria using mosquito nets impregnated with pyrethroids in Burkina Faso. Bull. Soc. Pathol. Exot. Filiales 81, 832-846 [in French].

Charlwood, J.D., Smith, T., Kihonda, J., Heiz, B., Billingsley, P.F., Takken, W., 1995a. Density independent feeding success of malaria vectors (Diptera: Culicidae) in Tanzania. Bull. Entomol. Res. 85, 29-35.

Charlwood, J.D., Kihonda, J., Sama, S., Billingsley, P.F., Hadji, H., Verhave, J.P., Lyimo, E., Luttikhuizen, P.C., Smith, T., 1995b. The rise and fall of Anopheles arabiensis (Diptera: Culicidae) in a Tanzanian village. Bull. Entomol. Res. 85, 37-44.

Charlwood, J.D., Smith, T., Billingsley, P.F., Takken, W., Lyimo, E.O.L., Meuwissen, J.H.E.T., 1997. Survival and infection probabilities of anthropophagic anophelines from an area of high prevalence of Plasmodium falciparum in humans. Bull. Entomol. Res. 87, 445-453.

Charlwood, J.D., Smith, T., Lyimo, E., Kitua, A.Y., Masanja, H., Booth, M., Alonso, P.L., Tanner, M., 1998. Incidence of Plasmodium falciparum infection in infants in relation to exposure to sporozoite-infected anophelines. Am. J. Trop. Med. Hyg. 59, 243-251.

Charlwood, J.D., Qassim, M., Elnsur, E.I., Donnelly, M., Petrarca, V., Billingsley, P.F., Pinto, J., Smith, T., 2001. The impact of indoor residual spraying with malathion on malaria in refugee camps in eastern Sudan. Acta Trop. 80, 1-8.

Curtis, C.F., Mnzava, A.E., 2000. Comparison of house spraying and insecticide-treated nets for malaria control. Bull. World Health Organ. 78, 1389-1400.

Curtis, C.F., Lines, J.D., Ijumba, J., Callaghan, A., Hill, N., Karimzad, M.A., 1987. The relative efficacy of repellents against mosquito vectors of disease. Med. Vet. Entomol. 1, 109-119.

Curtis, C.F., Myamba, J., Wilkes, T.J., 1996. Comparison of different insecticides and fabrics for anti-mosquito bednets and curtains. Med. Vet. Entomol. 10, 1-11.

Curtis, C., Maxwell, C., Lemnge, M., Kilama, W.L., Steketee, R.W., Hawley, W.A., Bergevin, Y., Campbell, C.C., Sachs, J., Teklehaimanot, A., Ochola, S., Guyatt, H., Snow, R.W., 2003. Scaling-up coverage with insecticide-treated nets against malaria in Africa: who should pay? Lancet Infect. Dis. 3, 304-307.

Dye, C., Hasibeder, G., 1986. Population dynamics of mosquitoborne disease: effects of flies which bite some people more frequently than others. Trans. R. Soc. Trop. Med. Hyg. 80, 69-77.

Erlanger, T.E., Enayati, A.A., Hemingway, J., Mshinda, H., Tami, A., Lengeler, C., 2004. Field issues related to effectiveness of insecticide-treated nets in Tanzania. Med. Vet. Entomol. 18, 153-160.

Fanello, C., Carneiro, I., Ilboudo-Sanogo, E., Cuzin-Ouattara, N., Badolo, A., Curtis, C.F., 2003. Comparative evaluation of carbosulfan- and permethrin-impregnated curtains for preventing house-entry by the malaria vector Anopheles gambiae in Burkina Faso. Med. Vet. Entomol. 17, 333-338.

Genton, B., Hii, J., al-Yaman, F., Paru, R., Beck, H.P., Ginny, M., Dagoro, H., Lewis, D., Alpers, M.P., 1994. The use of untreated bednets and malaria infection, morbidity and immunity. Ann. Trop. Med. Parasitol. 88, 263-270.

Gillies, M.T., 1954. Studies in house-leaving and outside resting of Anopheles gambiae Giles and Anopheles funestus Giles in East Africa. Bull. Entomol. Res. 45, 375-387.

Gillies, M.T., Coetzee, M., 1987. A Supplement to the Anophelinae of Africa South of the Sahara (Afrotropical Region). South African Medical Research Institute, Johannesburg.
Gillies, M.T., DeMeillon, B., 1968. The Anophelinae of Africa South of the Sahara (Ethiopian Zoogeographical Region). South African Institute for Medical Research, Johannesburg.

Gillies, M.T., Wilkes, T.J., 1969. A comparison of the range of attraction of animal baits for some West African mosquitoes. Bull. Entomol. Res. 59, 441-456.

Gillies, M.T., Wilkes, T.J., 1970. The range of attraction of single baits for some West African mosquitoes. Bull. Entomol. Res. 60, 225-235.

Gillies, M.T., Wilkes, T.J., 1972. The range of attraction of animal baits and carbon dioxide for mosquitoes. Studies in a freshwater area of West Africa. Bull. Entomol. Res. 61, 389-404.

Gimnig, J.E., Kolczak, M.S., Hightower, A.W., Vulule, J.M., Schoute, E., Kamau, L., Phillips-Howard, P.A., ter Kuile, F.O., Nahlen, B.L., Hawley, W.A., 2003a. Effect of permethrin-treated bed nets on the spatial distribution of malaria vectors in western Kenya. Am. J. Trop. Med. Hyg. 68, 115-120.

Gimnig, J.E., Vulule, J.M., Lo, T.Q., Kamau, L., Kolczak, M.S., Phillips-Howard, P.A., Mathenge, E.M., ter Kuile, F.O., Nahlen, B.L., Hightower, A.W., Hawley, W.A., 2003b. Impact of permethrin-treated bed nets on entomologic indices in an area of intense year-round malaria transmission. Am. J. Trop. Med. Hyg. 68, 16-22.

Grabowsky, M., Nobiya, T., Ahun, M., Donna, R., Lengor, M., Zimmerman, D., Ladd, H., Hoekstra, E., Bello, A., Baffoe-Wilmot, A., Amofah, G., 2005. Distributing insecticide-treated bednets during measles vaccination: a low-cost means of achieving high and equitable coverage. Bull. World Health Organ. 83, 195201.

Graham, K., Kayedi, M.H., Maxwell, C., Kaur, H., Rehman, H., Malima, R., Curtis, C.F., Lines, J.D., Rowland, M.W., 2005. Multicountry field trials comparing wash-resistance of PermaNet and conventional insecticide-treated nets against anopheline and culicine mosquitoes. Med. Vet. Entomol. 19, 72-83.

Gu, W., Regens, J.L., Beier, J.C., Novak, R.J., 2006. Source reduction of mosquito larval habitats has unexpected consequences on malaria transmission. Proc. Natl. Acad. Sci. USA 103, 17560-17563.

Haines, A., Cassels, A., 2004. Can the millennium development goals be attained? BMJ 329, 394-397.

Hasibeder, G., Dye, C., 1988. Population dynamics of mosquitoborne disease: persistence in a completely heterogeneous environment. Theor. Popul. Biol. 33, 31-53.

Hawley, W.A., Phillips-Howard, P.A., ter Kuile, F.O., Terlouw, D.J., Vulule, J.M., Ombok, M., Nahlen, B.L., Gimnig, J.E., Kariuki, S.K., Kolczak, M.S., Hightower, A.W., 2003. Community-wide effects of permethrin-treated bednets on child mortality and malaria morbidity in western Kenya. Am. J. Trop. Med. Hyg. 68 (Suppl. 4), 121-127.

Henry, M.C., Assi, S.B., Rogier, C., Dossou-Yovo, J., Chandre, F., Guillet, P., Carnevale, P., 2005. Protective efficacy of lambdacyhalothrin treated nets in Anopheles gambiae pyrethroid resistance areas of Cote d'Ivoire. Am. J. Trop. Med. Hyg. 73, 859-864.

Hewitt, S., Ford, E., Urhaman, H., Muhammad, N., Rowland, M., 1997. The effect of bednets on unprotected people: open-air studies in an Afghan refugee village. Bull. Entomol. Res. 87, 455-459.

Hii, J.L.K., Smith, T., Vounatsou, P., Alexander, N., Mai, A., Ibam, E., Alpers, M.P., 2001. Area effects of bednet use in a malariaendemic area in Papua New Guinea. Trans. R. Soc. Trop. Med. Hyg. 95, 7-13.

Howard, S.C., Omumbo, J., Nevill, C.G., Some, E.S., Donnelly, C.A., Snow, R.W., 2000. Evidence for a mass community effect of insecticide-treated bednets on the incidence of malaria on the Kenyan coast. Trans. R. Soc. Trop. Med. Hyg. 94, 357-360.

Kawaguchi, I., Sasaki, A., Mogi, M., 2004. Combining zooprophylaxis and insecticide spraying: a malaria-control strategy limiting 
the development of insecticide resistance in vector mosquitoes. Proc. R. Soc. Lond. B Biol. Sci. 271, 301-309.

Kelly, D.W., Thompson, C.E., 2000. Epidemiology and optimal foraging: modeling the ideal free distribution of insect vectors. Parasitology 120, 319-327.

Killeen, G.F., McKenzie, F.E., Foy, B.D., Schieffelin, C., Billingsley, P.F., Beier, J.C., 2000a. The potential impacts of integrated malaria transmission control on entomologic inoculation rate in highly endemic areas. Am. J. Trop. Med. Hyg. 62, 545-551.

Killeen, G.F., McKenzie, F.E., Foy, B.D., Schieffelin, C., Billingsley, P.F., Beier, J.C., 2000b. A simplified model for predicting malaria entomologic inoculation rates based on entomologic and parasitologic parameters relevant to control. Am. J. Trop. Med. Hyg. 62, 535-544.

Killeen, G.F., McKenzie, F.E., Foy, B.D., Bøgh, C., Beier, J.C., 2001. The availability of potential hosts as a determinant of feeding behaviours and malaria transmission by mosquito populations. Trans. R. Soc. Trop. Med. Hyg. 95, 469-476.

Killeen, G.F., Seyoum, A., Knols, B.G.J., 2004. Rationalizing historical successes of malaria control in Africa in terms of mosquito resource availability management. Am. J. Trop. Med. Hyg. 71 (Suppl. 2), 87-93.

Killeen, G.F., Ross, A., Smith, T.A., 2006a. Infectiousness of malariaendemic human populations to vector mosquitoes. Am. J. Trop. Med. Hyg. 76 (Suppl. 2), 38-45.

Killeen, G.F., Kihonda, J., Lyimo, E., Okech, F.R., Kotas, M.E., Mathenge, E., Schellenberg, J., Lengeler, C., Smith, T.A., Drakeley, C., 2006b. Quantifying behavioural interactions between humans and mosquitoes: evaluating the protective efficacy of insecticidal nets against malaria transmission in rural Tanzania. BMC Infect. Dis. 6, 161.

Kitua, A.Y., Smith, T., Alonso, P.L., Masanja, H., Urassa, H., Menendez, C., Kimario, J., Tanner, M., 1996. Plasmodium falciparum malaria in the first year of life in an area of intense and perennial transmission. Trop. Med. Int. Health 1, 475-484.

Kouznetsov, R.L., 1977. Malaria control by application of indoor spraying of residual insecticides in tropical Africa and its impact on community health. Trop. Doct. 7, 81-93.

Lengeler, C., 2004a. Insecticide-treated bed nets and curtains for preventing malaria. Cochrane Database Syst. Rev. CD000363.

Lengeler, C., 2004b. Insecticide-treated nets for malaria control: real gains. Bull. World Health Organ. 82, 84.

Lindsay, S.W., Snow, R.W., Broomfield, G.L., Semega Janneh, M., Wirtz, R.A., Greenwood, B.M., 1989. Impact of permethrintreated bednets on malaria transmission by the Anopheles gambiae complex in The Gambia. Med. Vet. Entomol. 3, 263-271.

Lindsay, S.W., Adiamah, J.H., Miller, J.E., Armstrong, J.R.M., 1991. Pyrethroid-treated bednet effects on mosquitoes of the Anopheles gambiae complex. Med. Vet. Entomol. 5, 477-483.

Lindsay, S.W., Adiamah, J.H., Armstrong, J.R.M., 1992. The effect of permethrin-impregnated bed nets on house entry by mosquitoes in The Gambia. Bull. Entomol. Res. 82, 49-55.

Lindsay, S.W., Armstrong-Schellenberg, J.R.M., Zeiler, H.A., Daly, R.J., Salum, F.M., Wilkins, H.A., 1995. Exposure of Gambian children to Anopheles gambiae vectors in an irrigated rice production area. Med. Vet. Entomol. 9, 50-58.

Lines, J.D., Myamba, J., Curtis, C.F., 1987. Experimental hut trials of permethrin-impregnated mosquito nets and eave curtains against malaria vectors in Tanzania. Med. Vet. Entomol. 1, 37-51.

Lines, J., Lengeler, C., Cham, K., de Savigny, D., Chimumbwa, J., Langi, P., Carroll, D., Mills, A., Hanson, K., Webster, J., Lynch, M., Addington, W. , Hill, J., Rowland, M., Worrall, E., MacDonald, M., Kilian, A., 2003. Scaling-up and sustaining insecticidetreated net coverage. Lancet Infect. Dis. 3, 465-466.

Mabaso, M.L., Sharp, B., Lengeler, C., 2004. Historical review of malarial control in southern African with emphasis on the use of indoor residual house-spraying. Trop. Med. Int. Health 9 846-856.

Magesa, S.M., Wilkes, T.J., Mnzava, A.E.P., Njunwa, K.J., Myamba, J., Kivuyo, M.D.P., Hill, N., Lines, J.D., Curtis, C.F., 1991. Trial of pyrethroid impregnated bednets in an area of Tanzania holoendemic for malaria. Part 2. Effects on the malaria vector population. Acta Trop. 49, 97-108.

Magesa, S.M., Lengeler, C., deSavigny, D., Miller, J.E., Njau, R.J., Kramer, K., Kitua, A., Mwita, A., 2005. Creating an 'enabling environment' for taking insecticide treated nets to national scale: the Tanzanian experience. Malar. J. 4, 34.

Mathenge, E.M., Gimnig, J.E., Kolczak, M., Ombok, M., Irungu, L.W., Hawley, W.A., 2001. Effect of permethrin-impregnated nets on exiting behaviour, blood feeding success, and time of feeding of malaria mosquitoes (Diptera: Culicidae) in Western Kenya. J. Med. Entomol. 38, 531-536.

Maxwell, C.A., Msuya, E., Sudi, M., Njunwa, K.J., Carneiro, I.A., Curtis, C.F., 2002. Effect of community-wide use of insecticidetreated nets for 3-4 years on malarial morbidity in Tanzania. Trop. Med. Int. Health 7, 1003-1008.

Maxwell, C.A., Rwegoshora, T., Magesa, S.M., Curtis, C.F., 2006a. Comparison of coverage with insecticide-treated nets in a Tanzanian town and villages where nets and insecticide are either marketed or provided free of charge. Malar. J. 5, 44.

Maxwell, C.A., Myamba, J., Magoma, J., Rwegoshora, R.T., Magesa, S.M., Curtis, C.F., 2006b. Tests of Olyset nets by bioassay and in experimental huts. J. Vector Borne Dis. 43, 1-6.

Millennium Development Project, 2005. Final report to United Nations Secretary General. United Nations, London/Sterling, VA

Miller, J.E., Lindsay, S.W., Armstrong, J.R.M., 1991. Experimental hut trials of bednets impregnated with synthetic pyrethroid and organophosphate insecticides for mosquito control in The Gambia. Med. Vet. Entomol. 5, 465-476.

Muirhead-Thomson, R.C., 1960. The significance of irritability, behaviouristic avoidance and allied phenomena in malaria erad ication. Bull. World Health Organ. 22, 721-734.

Mushi, A.K., Schellenberg, J.R., Mponda, H., Lengeler, C., 2003. Targeted subsidy for malaria control with treated nets using a discount voucher system in Tanzania. Health Policy Plan. 18, 163-171.

Pennetier, C., Corbel, V., Hougard, J.M., 2005. Combination of a non-pyrethroid insecticide and a repellent: a new approach for controlling knockdown-resistant mosquitoes. Am. J. Trop. Med. Hyg. 72, 739-744.

Pleass, R.J., Armstrong, J.R.M., Curtis, C.F., Jawara, M., Lindsay, S.W., 1993. Comparison of permethrin treatments for bednets in The Gambia. Bull. Entomol. Res. 83, 133-140.

Potikasikorn, J., Chareonviriyaphap, T., Bangs, M.J., Prabaripai, A., 2005. Behavioral responses to DDT and pyrethroids between Anopheles minimus species A and C, malaria vectors in Thailand. Am. J. Trop. Med. Hyg. 73, 343-349.

Robert, V., Carnevale, P., 1991. Influence of deltamethrin treatment of bed nets on malaria transmission in the Kou valley, Burkina Faso. Bull. World Health Organ. 69, 735-740.

Roberts, D.R., Andre, R.G., 1994. Insecticide resistance issues in vector-borne disease control. Am. J. Trop. Med. Hyg. 50, 21-43.

Roberts, D.R., Alecrim, W.D., Hshieh, P., Grieco, J.P., Bangs, M., Andre, R.G., Chareonviriphap, T., 2000. A probability model of vector behavior: effects of DDT repellency, irritancy, and toxicity in malaria control. J. Vector Ecol. 25, 48-61.

Roll Back Malaria Partnership, 2005a. World Malaria Report. World Health Organization, Geneva.

Roll Back Malaria Partnership, 2005b. Scaling up insecticide treated netting programmes in Africa: a strategic framework for coordinated national action. World Health Organization, Geneva.

Rowland, M., Durrani, N., Kenward, M., Mohammed, N., Urahman, H., Hewitt, S., 2001. Control of malaria in Pakistan by apply- 
ing deltamethrin insecticide to cattle: a community-randomised trial. Lancet 357, 1837-1841.

Rozendaal, J.A., 1997. Vector Control. Methods for use by individuals and communities. World Health Organization, Geneva.

Saul, A., 2003. Zooprophylaxis or zoopotentiation: the outcome of introducing animals on vector transmission is highly dependent on the mosquito mortality while searching. Malar. J. 2, 32.

Schellenberg, J.R., Abdulla, S., Nathan, R., Mukasa, O., Marchant, T.J., Kikumbih, N., Mushi, A.K., Mponda, H., Minja, H., Mshinda, H., Tanner, M., Lengeler, C., 2001. Effect of large-scale social marketing of insecticide-treated nets on child survival in rural Tanzania. Lancet 357, 1241-1247.

Smith, D.L., McKenzie, F.E., 2004. Statics and dynamics of malaria infection in Anopheles mosquitoes. Malar. J. 3, 13.

Smith, T., Charlwood, J.D., Kihonda, J., Mwankusye, S., Billingsley, P., Meuwissen, J., Lyimo, E., Takken, W., Teuscher, T., Tanner, M., 1993. Absence of seasonal variation in malaria parasitemia in an area of intense seasonal transmission. Acta Trop. 54, 55-72.

Smith, T., Charlwood, J.D., Takken, W., Tanner, M., Spiegelhalter, D.J., 1995. Mapping densities of malaria vectors within a single village. Acta Trop. 59, 1-18.

Smith, T., Charlwood, J.D., Kitua, A.Y., Masanja, H., Mwankusye, S., Alonso, P.L., Tanner, M., 1998. Relationship of malaria morbidity with exposure to Plasmodium falciparum in young children in a highly endemic area. Am. J. Trop. Med. Hyg. 59, 252-257.

Smith, T.A., Leuenberger, R., Lengeler, C., 2001. Child mortality and malaria transmission intensity in Africa. Trends Parasitol. 17, 145-149.

Snow, R.W., Guerra, C.A., Noor, A.M., Myint, H.Y., Hay, S.I., 2005. The global distribution of clinical episodes of Plasmodium falciparum malaria. Nature 434, 214-217.
Soremekun, S., Maxwell, C., Zuwakuu, M., Chen, C., Michael, E., Curtis, C., 2004. Measuring the efficacy of insecticide treated bednets: the use of DNA fingerprinting to increase the accuracy of personal protection estimates in Tanzania. Trop. Med. Int. Health 9, 664-672.

Tami, A., Mubyazi, G., Talbert, A., Mshinda, H., Duchon, S., Lengeler, C., 2004. Evaluation of Olyset insecticide-treated nets distributed seven years previously in Tanzania. Malar. J. 3, 19.

Webster, J., Lines, J., Armstrong-Schellenberg, J.R.M., Hanson, K., 2005. Which delivery systems reach the poor: a review of equity of coverage of ever-treated nets, never-treated nets and immunization to reduce childhood mortality in Africa. Lancet Infect. Dis. 5, 709-717.

White, G.B., 1974. The Anopheles gambiae complex and disease transmission in Africa. Trans. R. Soc. Trop. Med. Hyg. 68, 279-301.

White, G.B., Magayuka, S.A., Boreham, P.F.L., 1972. Comparative studies on sibling species of the Anopheles gambiae Giles complex (Dipt., Culicidae): bionomics and vectorial activity of species A and species B at Segera, Tanzania. Bull. Entomol. Res. 62, 295-317.

Woolhouse, M.E.J., Dye, C., Etard, J.F., Smith, T., Charlwood, J.D., Garnett, G.P., Hagan, P., Hii, J.L.K., Ndhlovu, P.D., Quinnell, R.J., Watts, C.H., Chaniawana, S.K., Anderson, R.M., 1997. Heterogeneities in the transmission of infectious agents: implications for the design of control programs. Proc. Natl. Acad. Sci. USA 94, 338-342.

Yates, A., N'Guessan, R., Kaur, H., Akogbeto, M., Rowland, M., 2005. Evaluation of KO-Tab 1-2-3: a wash-resistant 'dip-it-yourself' insecticide formulation for long-lasting treatment of mosquito nets. Malar. J. 4, 52. 